\title{
MONTE CARLO SIMULATIONS OF ENERGY RESOLUTION OF THE ELECTROMAGNETIC CALORIMETER PROTOTYPE FOR ELECTRON-ION COLLIDER
}

\author{
A. A. HOGHMRTSYAN
}

A. Alikhanyan National Laboratory (AANL), Yerevan Physics Institute, Armenia

In this article, we present energy resolution studies of an electromagnetic calorimeter prototype for Electron-Ion Collider. The results of energy resolution for various configurations of lead tungstate crystals were obtained based on the Geant 4 simulation package. The energy resolution was studied as a function of the polar angle of incident electrons in a momentum range of 1 to $10 \mathrm{GeV}$.

https://doi.org/10.46991/PYSU:A/2021.55.1.090

Keywords: Electron Ion Collider, calorimetry, detector simulation.

Introduction. An Electron-Ion Collider (EIC) is a particle accelerator designed to collide an electron beam with a proton or nuclei to study the internal structure of those particles [1]. The project of EIC has been approved by the US department of energy, and is planned to be built over the next ten years at Brookhaven National Laboratory (BNL) in Upton, New York, with an estimated cost of \$1.6 billion to \$2.6 billion.

In the decades since the discovery of quarks, experiments in nuclear and particle physics have led to the fundamental theory of strong interactions - Quantum Chromodynamics (QCD that describes the extraordinary richness of nature at the subatomic level. Understanding how the properties of matter originate from the deeply fundamental constituents of QCD is the primary goal of nuclear physics and the central motivation for polarized electron-proton and electron-ion collisions at the EIC [1,2].

The basic scientific requirements for EIC are:

- highly polarized ( 70\%) beams of electrons, protons, and light ions (d, 3He);

- ion beams from deuteron to the heaviest nuclei (Uranium or Lead);

- variable center of mass energies from $\sim 20$ to $140 \mathrm{GeV}$;

- high collision luminosity of $\sim 10^{33-34} \mathrm{~cm}^{-2} \mathrm{~s}^{-1}$.

\footnotetext{
* E-mail: artur.hoghmrtsyan@gmail.com
} 
Proposed experimental setups that would operate on EIC are complex systems of various types of detectors (tracking, particle identification, energy and time measurement, etc.). Electromagnetic calorimeters are critical sub-detectors in high energy physics experiments [3]. The general idea behind calorimetry is to measure the energy of a particle as it passes through the detector. It is designed to stop or absorb most of the particles coming from a collision. Calorimeters consist of absorbing high-density material. Electromagnetic calorimeters measure the energy of electrons and photons as they interact with matter producing electromagnetic showers. The summed ionization is proportional to the energy deposited in the material and is a good measure of the incident energy.

A typical electromagnetic calorimeter is a light-transparent, homogeneous crystal large enough to contain the complete shower of secondary particles. Crystal calorimeters have been used in nuclear and high energy physics for their high energy resolution and detection efficiency.

The EIC high-resolution electromagnetic calorimeters have the following basic requirements:

- interaction rate capability up to $0.5 \cdot 10^{6} \mathrm{~Hz}$ requiring reasonably fast scintillation kinetics;

- sufficient energy resolution and efficiency over a large dynamic range of photon energies, typically from about $50 \mathrm{MeV}$ to $50 \mathrm{GeV}$;

- adapted geometrical dimensions to contain the major part of the electromagnetic shower;

- moderate radiation hardness up to $3 \mathrm{krad} /$ year $(30 \mathrm{~Gy} /$ year) for the electromagnetic and $10^{10} \mathrm{n} / \mathrm{cm}^{2}$ for the hadronic radiation at the maximal luminosity.

This precludes the use of most of the known scintillator materials. The most common choice to meet the above requirements is lead tungstate, $\mathrm{PbWO}_{4}(\mathrm{PWO}$ ), which features a small Moliere radius $\left(R_{M}=2.0 \mathrm{~cm}\right)$, high density $\left(8.3 \mathrm{~g} / \mathrm{cm}^{3}\right)$, fast response $(<2 \mathrm{~ns})$, and radiation resistance. $\mathrm{PbWO}_{4}$ has been used for existing calorimeters (CMS, JLab Hall B) [4,5], and high quality crystals are being considered to be used in several new electromagnetic calorimeter projects around the world (PANDA, JLab $12 \mathrm{GeV}$ ). Energy resolutions better than $2 \%$ for energies of $0.05-$ $15 \mathrm{GeV}$ have been achieved with the PANDA prototype with a constant term of the order of $0.6-0.7 \%$. CMS has achieved a constant term of $0.3 \%$, albeit at higher energies $(20-250 \mathrm{GeV})$. For comparison, the energy resolution of lead glass is $5-6 \%$. The energy resolution of $\mathrm{PbWO}_{4}$ can be further improved by cooling the crystals.

Geant4 Simulation and Results. Geant 4 is a software package designed for modeling the passage of particles through matter based on the Monte Carlo method [6]. This toolkit is a set of libraries implemented in C++. Configuration of particle types included in the simulation, physical processes, models of particle interactions, and their application boundaries are described in a particular class of program based on Geant4 PhysicsList. Usually, PhysicsList includes a set of electromagnetic and hadronic interactions, decay of nuclei and particles, as well as parameterized interac- 
tion models. In obtaining the results presented in this article, we used the FTFP-BERT PhysicsList [7].

The simulation of electromagnetic showers was carried out for three different configurations of matrices of $\mathrm{PbWO}_{4}$ crystals. The crystals dimensions were $20 \times$ $20 \times 200 \mathrm{~mm}$, with matrix sizes of $1 \times 1,3 \times 3$ and $5 \times 5$. The distribution of total energy deposited in the matrix was fited with Novosibirsk function in order to obtain the energy resolution. The choice of the function that is given in Eq. (1), was driven by the asymmetric tail of the distribution [8],

where

$$
f(x)=A \exp \left[-0.5\left(\frac{\ln ^{2}[1+\Lambda \tau(x-\mu)]}{\tau^{2}}+\tau^{2}\right)\right],
$$

$$
\Lambda \equiv \frac{\sinh (\tau \sqrt{\ln 4})}{\sigma \tau \sqrt{\ln 4}}
$$

Here, $\mu$ is the peak position, $\sigma$ is the width, and $\tau$ is a parameter describing the tail.

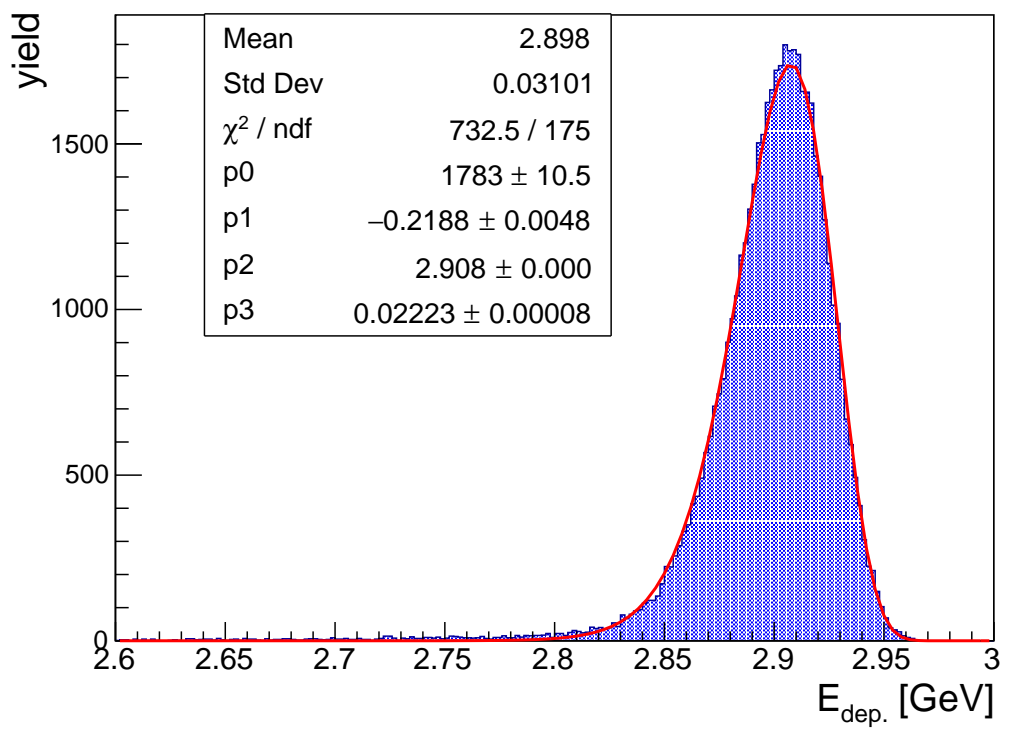

Fig. 1. Distribution of total energy deposition in $5 \times 5$ matrix of crystals for $3 \mathrm{GeV}$ incident electron beam sent to the central crystal, perpendicular to its surface.

An example of the spectra of the total deposited energy and the fit for $3 \mathrm{GeV}$ incident electron, which was directed to the center of $5 \times 5$ matrix, is shown in Fig. 1 . The energy dependence of the mean and the width of the deposited energy distribution as a functions of incident electron momentum are shown on Fig. 2, for three different matrix configurations $(1 \times 1,3 \times 3$ and $5 \times 5)$. The incident electron was sent to the center of the matrix perpendicular to the surface of the crystals. The energy dependence of the resolution is well described by the function:

$$
\frac{\sigma}{E}=\frac{a}{\sqrt{E}}+b
$$


where $a$ is the stochastic term and $b$ is the constant term. The results obtained for the parameter $a$ are $3.559,1.598$ and $0.901 \%$ for three different matrix configurations and show a clear decrease with the increase of the matrix dimensions. The constant term varies within the range of $0.160-0.231 \%$.
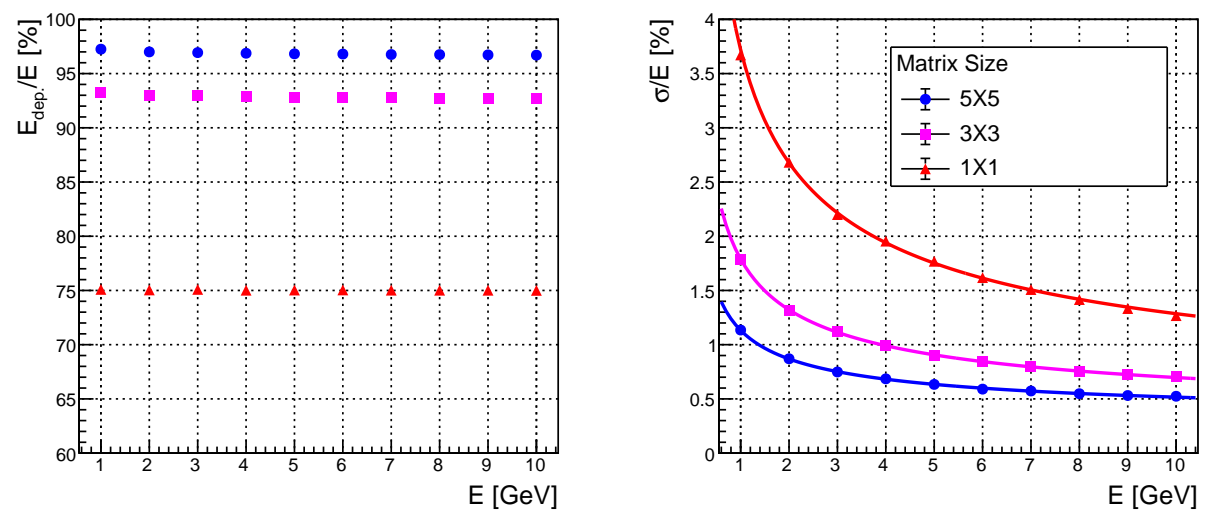

Fig. 2. Energy dependence of fractional energy deposition for three configurations of crystal matrices $1 \times 1,3 \times 3$ and $5 \times 5$ (left), and energy dependence of resolution for the same configuration of matrices (right).
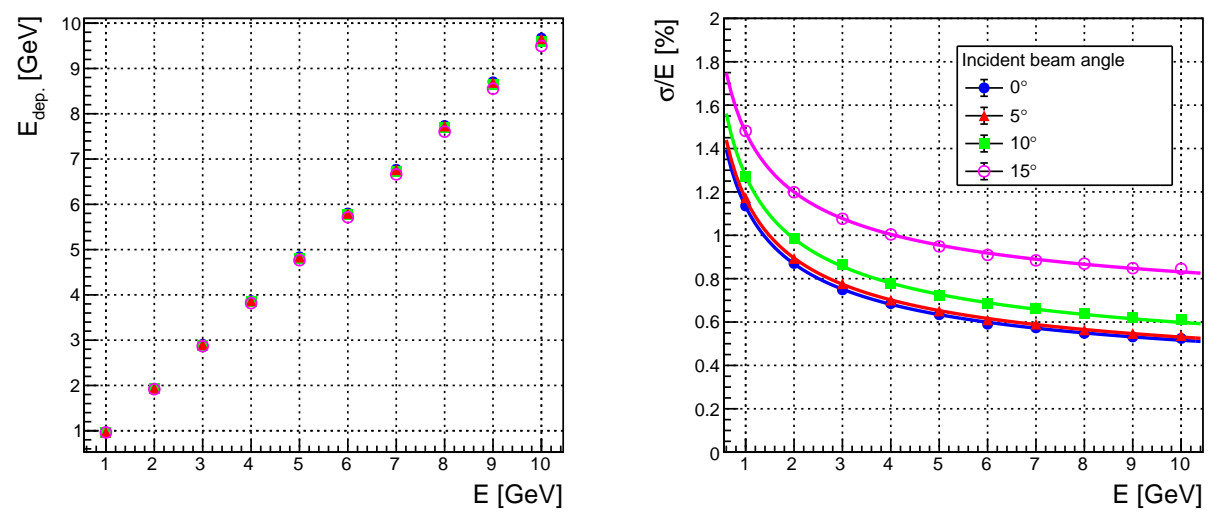

Fig. 3. Total energy deposition as a function of incident electron energy for different polar angles (left) and energy resolution for different polar angles of incident electrons (right).

Next, we investigated the effect of the polar angle of an incident electron on the energy resolution of the calorimeter prototype made of lead tungstate crystals. For this purpose, a Monte Carlo dataset was simulated for a $5 \times 5$ matrix of crystals with different polar angles for incident electrons. The left plot in Fig. 3 shows the total energy deposited into the matrix as a function of the incident electron energy for different polar angles. The decline of the total energy deposition with an increase in the polar angle is negligible, while the resolution parameters change significantly, as can be seen from the right plot in Fig. 3. The resolution parameters obtained for different values of polar angle of incident electrons are given in Table. 
Energy resolution parameters for different polar angles of incident electrons sent to the center of central crystal of the $5 \times 5$ matrix

\begin{tabular}{|c|c|c|}
\hline Angle of electrons & Parameter $a, \%$ & Parameter $b, \%$ \\
\hline $0^{\circ}$ & $0.901 \pm 0.005$ & $0.231 \pm 0.002$ \\
\hline $5^{\circ}$ & $0.932 \pm 0.006$ & $0.236 \pm 0.003$ \\
\hline $10^{\circ}$ & $0.941 \pm 0.006$ & $0.286 \pm 0.004$ \\
\hline $15^{\circ}$ & $0.988 \pm 0.006$ & $0.534 \pm 0.004$ \\
\hline
\end{tabular}

Conclusion. Based on Geant4 simulations, we studied the performance of the electromagnetic calorimeter prototype made of lead tungstate $\mathrm{PbWO}_{4}$ crystals as a possible option for upcoming EIC experiments. The energy resolution of such a calorimeter was evaluated as a function of the polar angle of an incident electron, as well as for different dimensions of crystal matrices. The results obtained can help to estimate the optimal detector design for future experiments in EIC.

Received 12.03.2021

Reviewed 19.04.2021

Accepted 28.04.2021

\section{R E F E R E N C E S}

1. Accardi A. et al. Electron Ion Collider: The Next QCD Frontier: Understanding the Glue That Binds Us All. Eur. Phys. J. A 52 (2016), 268 p.

http://doi.org/10.1140/epja/i2016-16268-9

2. Aschenauer A.C. et al. Nuclear Structure Functions at a Future Electron-Ion Collider. Phys. Rev. D 96 (2017), 114005.

http://doi.org/10.1103/PhysRevD.96.114005

3. Fabjan C.W., Gianotti F. Calorimetry for Particle Physics. Rev. Mod. Phys. 75 (2003), $1243 \mathrm{p}$. http://doi.org/10.1103/RevModPhys.75.1243

4. CMS Collaboration “The Electromagnetic Calorimeter Project Technical Design Report”. CERN/LHCC/97-33 (1997).

5. Kubantsev M. et al. Performance of the PrimEx Electromagnetic Calorimeter. AIP Conf. Proc. 867 (2006), 51 p. http://doi.org/10.1063/1.2396938

6. Agostinelli S. et al. GEANT4 - a Simulation Toolkit. Nucl. Inst. Meth. A 506 (2003), 250p. http://doi.org/10.1016/S0168-9002(03)01368-8

7. Apostolakis J. et al. GEANT4 Physics Lists for HEP. IEEE Nucl. Sci. Symp. Conf. Rec. 2008 (2008), 833 p. 
8. Ikeda H. et al. A Detailed Test of the CsI(Tl) Calorimeter for BELLE with Photon Beams of Energy between $20 \mathrm{MeV}$ and $5.4 \mathrm{GeV}$. Nucl. Inst. Meth. A 441 (2000), 401-426.

http://doi.org/10.1016/S0168-9002(99)00992-4

\section{U. U. くกาบกร3ญไ}

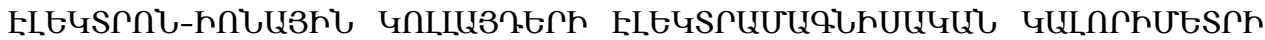

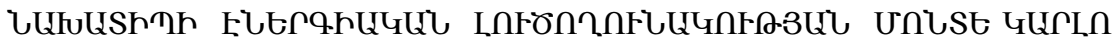

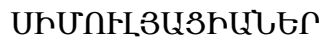

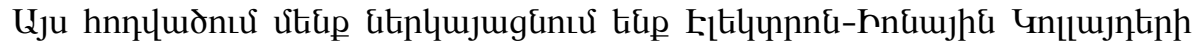

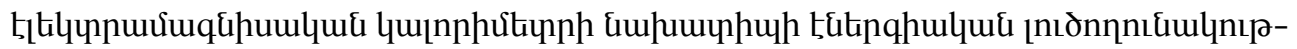

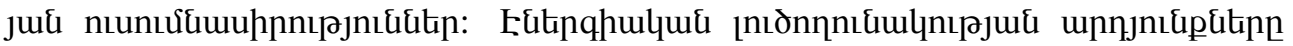

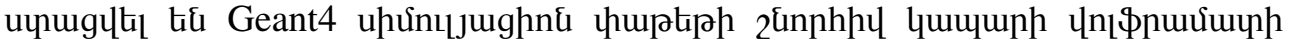

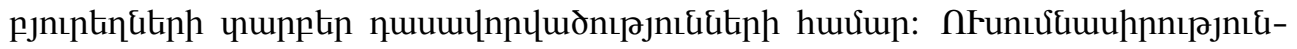

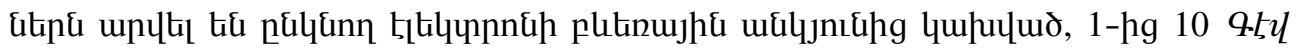

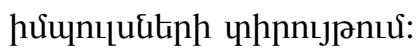

\section{А. А. ОГМРЦЯН}

\section{МОНТЕ-КАРЛО СИММУЛЯЦИИ ЭНЕРГЕТИЧЕСКОГО РАЗРЕШЕНИЯ ПРОТОТИПА ЭЛЕКТРОМАГНИТНОГО КАЛОРИМЕТРА ДЛЯ ЭЛЕКТРОННО-ИОННОГО КОЛЛАЙДЕРА}

В статье представлены исследования энергетического разрешения прототипа электромагнитного калориметра для электронно-ионного коллайдера. Результаты энергетического разрешения для различных конфигураций кристаллов вольфрамата свинца получены на основе пакета моделирования Geant4. Энергетическое разрешение исследовалось в зависимости от полярного угла падающих электронов в диапазоне импульсов 1-10 ГэВ. 